\title{
Albumin microparticles as the carriers for allopurinol and applicable for the treatment of ischemic stroke
}

\author{
Hovsep Alexandr Aganyants ${ }^{1}$ - Gayane Nikohosyan ${ }^{1,2} \cdot$ Kristine Edgar Danielyan $^{1}$
}

Received: 7 April 2015/Accepted: 18 September 2015/Published online: 9 November 2015

(C) The Author(s) 2015. This article is published with open access at Springerlink.com

\begin{abstract}
Albumin nanoparticles are already used for the treatment of the cancer. In our current work, it is presented the technique for the preparation of small-size 1- to 5-micron particles coated with the allopurinol. We propose that this combination of the compounds might be useful for the ischemic stroke treatment as the agent preventing formation of the brain edema, reactive oxygen species, and initiation of cells regeneration. Glutaraldehyde was used for the polymerization of albumin. Determination of the particle size was performed by the light as well as phase contrast microscopies and analyzed by Pixcavator 6.0 and Image Tool programs. Modification and establishment of iodine-based method served as the base for quantification of bound with the particles and free allopurinol. As a consequence of the experiments, the best formulation of glutaraldehyde ratio and albumin quantity as well as conditions for the formation of the smallest sized spheroidshaped particles were found for the further in vivo application.
\end{abstract}

Keywords Albumin - Particles - Allopurinol - Cancer . Stroke

$\begin{array}{ll}\text { Abbreviations } \\ \text { ROS } & \begin{array}{l}\text { Reactive oxygen } \\ \text { species }\end{array} \\ \text { GA } & \text { Glutaraldehyde }\end{array}$

Kristine Edgar Danielyan

kristine_danielyan@biochem.sci.am

1 H. Buniatian Institute of Biochemistry, National Academy of Science, RA, 5/1 Paruyr Sevak street, 0014 Yerevan, Armenia

2 Yerevan State University, Yerevan, Armenia

\section{Introduction}

In blood plasma, albumin is one of the multifunctional proteins which is found abundantly, $35-50 \mathrm{mg} / \mathrm{ml}$ [1]. Human serum albumin has a molecular weight equal to $66.348 \mathrm{kDa}[2,3]$. Human albumin consists of three domains: I, II, and III. Each domain has A and B subdomains. Crystallographic analysis of HSA has established that this protein contains $67 \% \alpha$-helical and no $\beta$-sheet [3]. The remaining structure, $33 \%$, consisting of the polypeptidic loop part, which makes the link between $\mathrm{A}$ and $\mathrm{B}$ subdomains, is as long as 20 amino acids. The helixes are locked together by 17 disulfide bonds, which, as believed, guarantee a thermal stability of the protein [4]. Fatty acid binding to HSA induces large-scale changes in the protein conformation, rotating domains I and III relative to domain II [4-6].

Based on Sudlow's early work, it was shown that drugs bind to two primary sites in subdomains, namely, IIA and IIIA $[2,7]$. These sites are similar and contain six helixes [4]. However, recent work showed that the steroid antibiotic, fusidic acid binds specifically to subdomain IB [8].

The human albumin gene is located on the long arm of chromosome 4 at position q13.3 [3].

Albumin might be synthesized in the liver and its total amount is $13.9 \mathrm{mg} / \mathrm{day}$ [1]. The main function of the albumin is to support osmotic pressure [1]. This protein binds to various endogenous compounds including longchain fatty acids, steroids, and L-tryptophan [9]. Albumin can also serve as a transporting agent for the delivery of copper, zinc, and calcium ions [1]. This plasma protein is able to carry medicines such as warfarin, ibuprofen, chlorpromazine, and naproxen. Binding of the compounds to albumin changes their targeting effects as well as the 
circulation time [10]. Consequently, albumin might serve as a carrier not only for the medicines but also for the toxins, for instance bilirubin [1].

Compared to synthetic polymers, proteins possess several advantages: They might be degraded into the peptides by naturally occurring enzymes; in comparison with the chemically synthesized nanomolecules, they may accumulate in the body and result in toxic degradation products [11]. Electrostatic interactions, hydrophobic attractions, and covalent bindings are the mechanisms allowing attaching the drug with the carrier. Also, these nanoparticles are able to be modified and present the attached molecules to the surface of the targeted cells [11]. It was demonstrated that uptake of albumin-paclitaxel nanoparticles is presumably mediated by the gp60 transcytosis pathway and subsequent binding to secreted protein, acidic and rich in cysteine (SPARC) in the tumor extracellular matrix [12].

In our current work, we are presenting the method for the formation of the albumin particles, which are coated with the allopurinol solution.

Allopurinol is one of the classical inhibitors of Xanthine Oxidase (XO), and one of the last enzymes of purines catabolism responsible for the formation of uric acid. It has a wide utility for the treatment of podagra. The by-products of XO activity are the reactive oxygen species (ROS). Recently, allopurinol was used in the clinical trial with involvement of the stroke patients. The published results of these trials are contradictive [13, 14].

We think that albumin nanoparticles carrying allopurinol will not possess negative side effects, including triggering liver necrosis. Also, the frequency of the treatment after utility of the allopurinol-coated particles in comparison with the free allopurinol will be less, which will improve the efficiency of this medicine. Allopurinol is metabolized by the liver with the $t_{1 / 2}$ equal to $1-2 \mathrm{~h}$. Binding with the albumin particles will prolong circulation time of this medicine and, consequently, will reduce the metabolic rate and prolong time of its gradual influence without necessity of second dosage uptake.

\section{Materials and methods}

\section{Preparation of the nanoparticles}

Different concentrations of serum albumin (Sigma) were dissolved in $1 \mathrm{ml}$ of water. Numerous concentrations of glutaraldehyde and $14 \mathrm{ml}$ of ethyl alcohol were added (GA; $25 \%$, Medisar, Armenia). We have used 20, 200, and $500 \mathrm{mg}$ of the albumin for the formation of the particles as well as 50, 500, and $2000 \mu \mathrm{l}$ of glutaraldehyde to be able to delineate the best composition for the formation of the small, spheroid-type particles. This mixture was incubated for $24 \mathrm{~h}$. Also, these reactions were carried out in water vs ethanol solution. The mixture was centrifugated at 8000 RPM for $15 \mathrm{~min}$. The precipitate was dissolved in $3 \mathrm{ml}$ of water, and the particles were washed from the remaining parts of the glutaraldehyde. The remaining part of the precipitate was dissolved in $400 \mu \mathrm{l}$ of $1 \times$ PBS [15-19]. For better visualization, when the formation of particles was completed, they were mixed with $2 \mathrm{ml}$ of $2 \%$ solution of Evans Blue (Sigma).

\section{Microscopy}

The work was performed with the utility of light microscopy as well as phase contrast lenses system with $100 \times$ of objective magnification (Binocular Biological Microscope, Model BM-180///SP, Germany). The number of the particles as well as the size of the formed albumin polymers was assessed with the Pixcavator 6.0 as well as Image Tool programs.

\section{Determination of the bound allopurinol with the particles}

The method is based on the ability of the iodine ethylene solution $(3 \%)$ to oxidize allopurinol and to form the precipitate. The spectrophotometric method was developed, and absorption was measured at $480 \mathrm{~nm}$. Particle suspension $(50 \mu \mathrm{l})$ was dissolved in $910 \mu \mathrm{l}$ of PBS $(1 \times)$, and $40 \mu \mathrm{l}$ of iodine solution was added $(20 \mu \mathrm{l}$ of $3 \%$ iodine was dissolved in $10 \mathrm{ml}$ PBS buffer). To measure the absorption, PerkinElmer Junior spectrophotometer was used [20].

\section{Iodometric oxidation reaction for calculation of allopurinol}

Purine derivatives such as the structures containing azo are able to form hardly dissolved complex compounds, which might be visualized by spectrophotometric techniques. The reaction was performed in acidic environment [21]. Twenty microliters of $3 \%$ ethanol iodine solution was dissolved in $10 \mathrm{ml}$ of buffer. As detecting agent, it was used $40 \mu \mathrm{l}$ of above mentioned iodine solution. The quantity of the allopurinol/1 $\mu \mathrm{l}$ was calculated after normalization with the particles containing precipitate or supernatants control solutions based on the following equation, where $\mathrm{X}$ the concentration and $\mathrm{Y}$ is the absorption.

$X=(Y / 1.657) / 50$

\section{Results}

1. Determination of the albumin particles size utilizing Image Tool program. 
The experiments were performed with three groups of particles. The total quantity of the protein, which was polymerizing, was equal to $200 \mathrm{mg}$. The analysis of the particles' size revealed that the group with the addition of $50 \mu \mathrm{l} \mathrm{GA}$ had a surface area equal to $62.946 \pm 2.697 \mu$, as well as $162.775 \pm 7.055 \mu$. Addition of $200 \mu \mathrm{l}$ of the GA provokes particles formation with surface area equal to $78.004 \pm 7.642$ and $221.992 \pm 39.859$. Addition of $500 \mu \mathrm{l}$ of GA results formation of the particles with the surface area equal to $62.449 \pm 2.431$ and $212.852 \pm 15.482 \mu$ (Fig. 1).

2. Determination of the formed particles number in the microscope field.

By adding of $2000 \mu \mathrm{l}$ of GA, the highest number of particles was formed and was equal to $23.3 \pm 7.93$. In 50- and 200- $\mu$ l GA groups, the number of the particles in the statistically significant way was different from the previously mentioned group and was equal to $5.7 \pm 1.136$ and $6.6 \pm 1.949$ (Fig. 1).

3. Formation of the particles in the presence of different concentration of the protein and determination of the number of formed particles (Fig. 2).

The number of the formed particles was evaluated after mild changes of the conditions of the experiment. After 24-h incubation, the mixture was centrifugated for $15 \mathrm{~min}, \quad \mathrm{RPM}=8000$. The precipitate was resuspended in water, and the samples were evaluated with the utility of light as well as phase contrast microscopy. The maximal number of the particles was observed in the samples with the addition of $20 \mathrm{mg}$ of the protein $699.00 \pm 35.87$; in the samples with the addition of $200 \mathrm{mg}$ of the albumin, the number was equal to $468.00 \pm 51.87$, whereas addition of $500 \mathrm{mg}$ resulted in the consequential formation of $288.76 \pm 17.83$ particles. It is quite interesting to mention that the shape of the particles was spheroid and did not look like a flake as it is shown in the Fig. 3. To have better visualization, the particles were stained with Evans Blue.

4. Formation of the particles in the presence of different concentration of the protein and determination of size of formed particles.

The size of the particles was gradually increasing with the increase in the concentration of the protein. In 20-mg samples the number was equal to $0.0155 \pm 0.001$, in $200 \mathrm{mg}-0.036 \pm 0.003$, and the largest particles were formed after addition of $500 \mathrm{mg}$ of protein $(0.056 \pm 0.003)$. The results of comparison of the groups were statistically significant.

We also compared the shape of the particles before centrifugation in water vs ethyl alcohol. In ethyl alcohol solution, the particles were more condensed
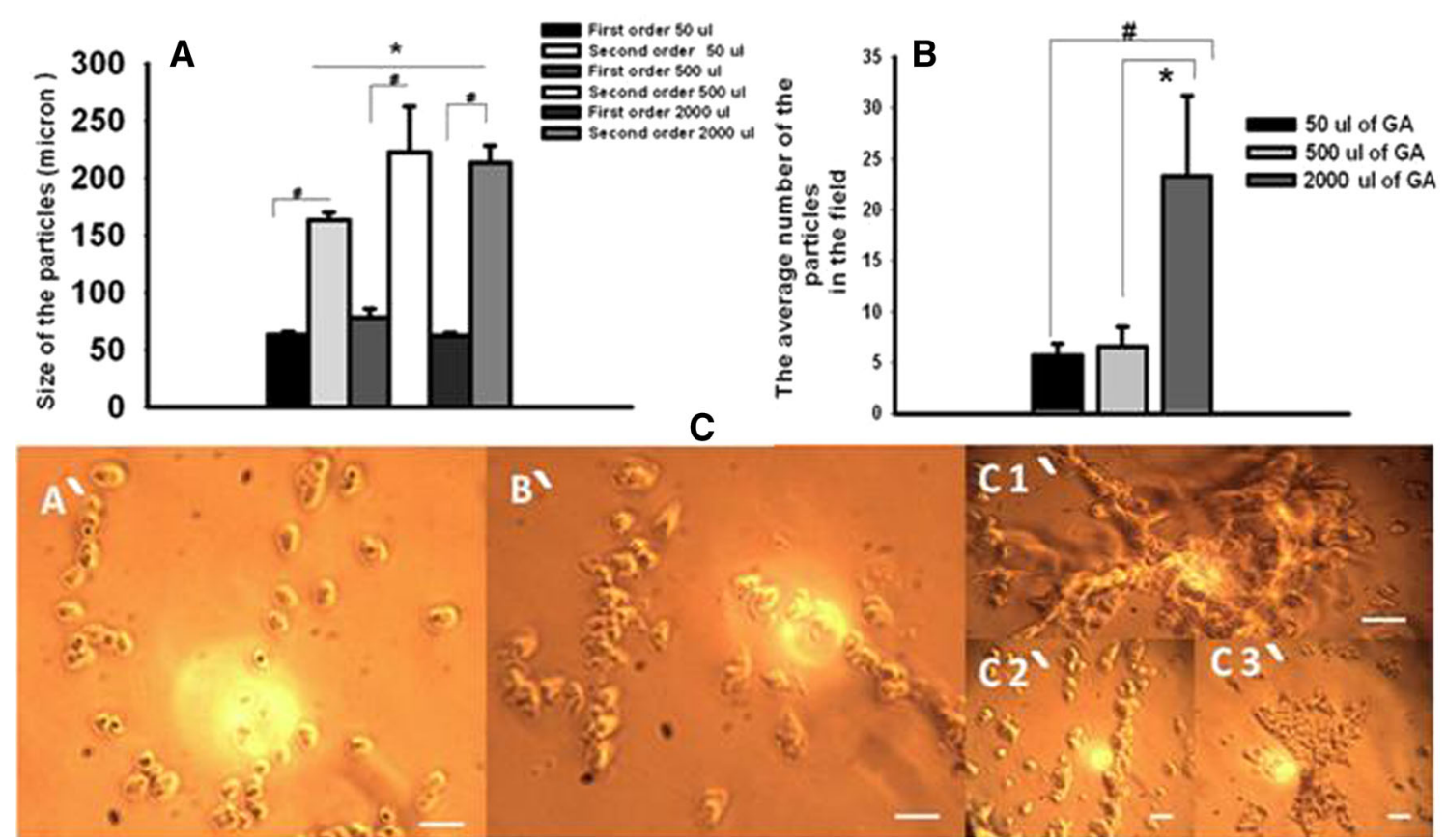

Fig. 1 Determination of the particle size after addition of different quantities of GA. After addition of $200 \mathrm{mg}$ into the mixture, we have incubated the particles for $24 \mathrm{~h}$. a The size of the particles, surface area, is presented as microns. The significance in the groups for 50, $500,2000 \mu \mathrm{l}$ of added GA was equal to $p=1.62283 \mathrm{E}-27$, $p=0.001327$ and $p=1.72321 \mathrm{E}-18$, respectively. The difference between the size of the large particle in the groups was $p=0.000304246$ and $p=0.003935424$, for $50 \mu \mathrm{l} \mathrm{GA}$ vs $500 \mu \mathrm{l}$ and 50 vs $2000 \mu \mathrm{l}$, respectively. We have used Image Tool program for the determination of particles sizes. b Representation of the pictures for $500 \mu \mathrm{l}$ and 50 vs $2000 \mu \mathrm{l} \mathrm{GA}$ containing groups (respectively they are the pictures $\mathbf{a}^{\prime}, \mathbf{b}^{\prime}$ and $\mathbf{c 1}^{\prime}+\mathbf{c 2}^{\prime}+\mathbf{c 3}^{\prime}$ 

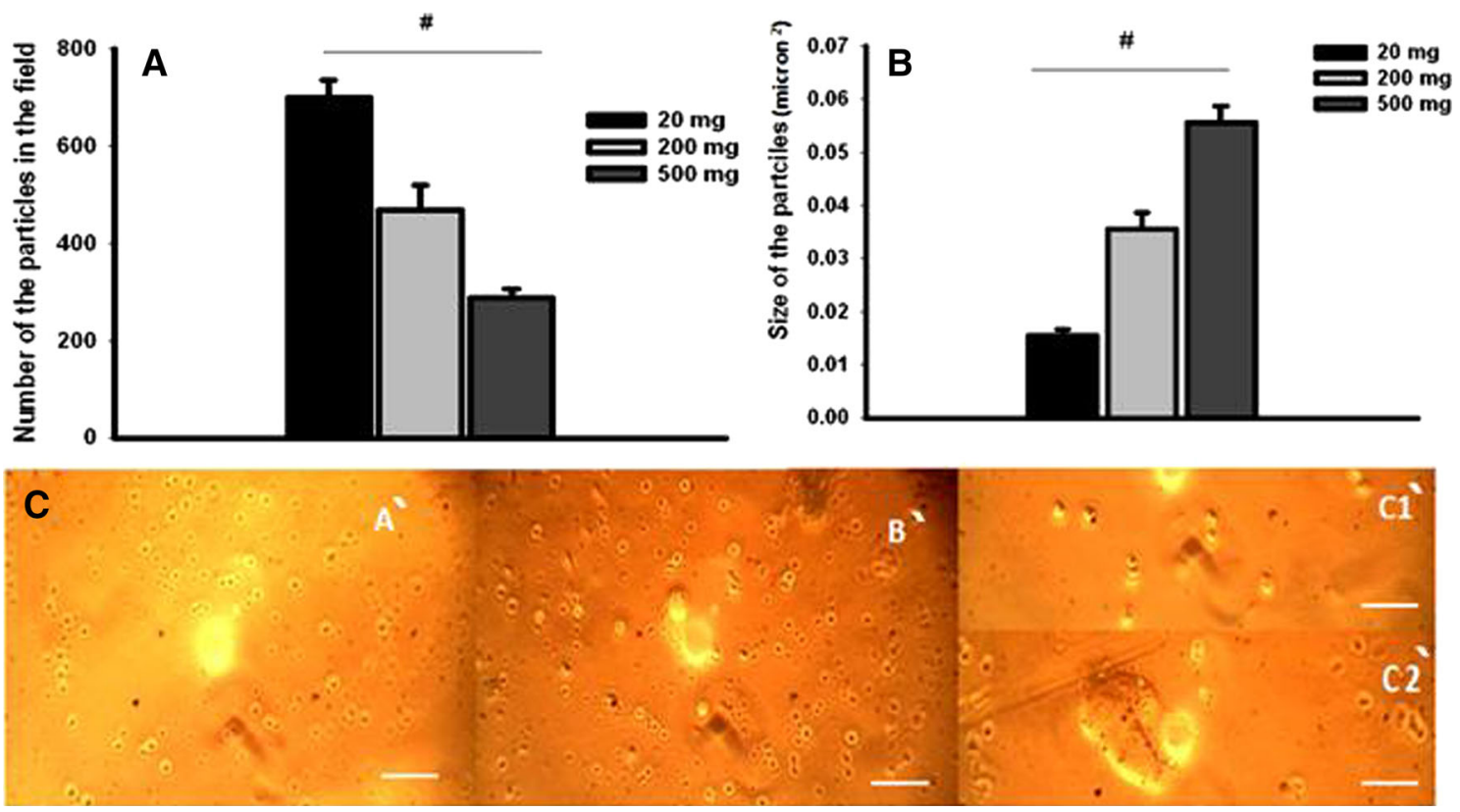

Fig. 2 Determination of the particle size after addition of different quantities of albumin. A. The number of the particles was calculated based on the micropictures and after utility of Pixcavator 6.0 program. The significances between groups for $20,200,500 \mathrm{mg}$ of added albumin, reflected by $p$, were lower than 0.05 (one-way ANOVA). b The size of the particles, surface area, is presented as the $\mu^{2}$. The significances between groups for $20,200,500 \mathrm{mg}$ of added albumin, reflected by $\mathrm{p}$, were lower than 0.05 (one-way ANOVA). c Representation of the pictures for $20,200,500 \mathrm{mg}$ of albumin-containing groups (respectively they are the pictures $\mathbf{a}^{\prime}, \mathbf{b}^{\prime}$ and $\mathbf{c 1}^{\prime}+\mathbf{c 2}^{\prime}$ )

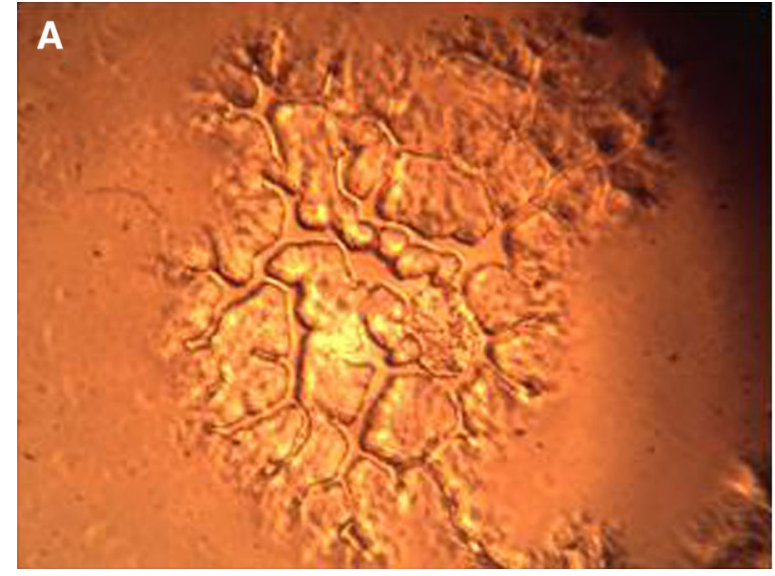

Fig. 3 Comparison of the particle shapes incubated in water vs ethanol. In $\mathbf{a}$ is represented the picture of the particle in water solution before centrifugation and, consequently, before formation of the

than in water solution. Thus, for the better coupling with the allopurinol, it was decided to use water instead of alcohol (Fig. 3).

5. It was also determined the quantity of allopurinol in 20-mg albumin-containing samples.

We worked with DMSO vs water solutions of allopurinol with the concentration of $0.07 \mathrm{mg} / \mathrm{ml}$. We have added 200, 400, 800 and $2000 \mu \mathrm{l}$ of allopurinol solution for the incubation with the particles. Also, we evaluated the effectiveness of the binding normalized with the equal amount of allopurinol stock

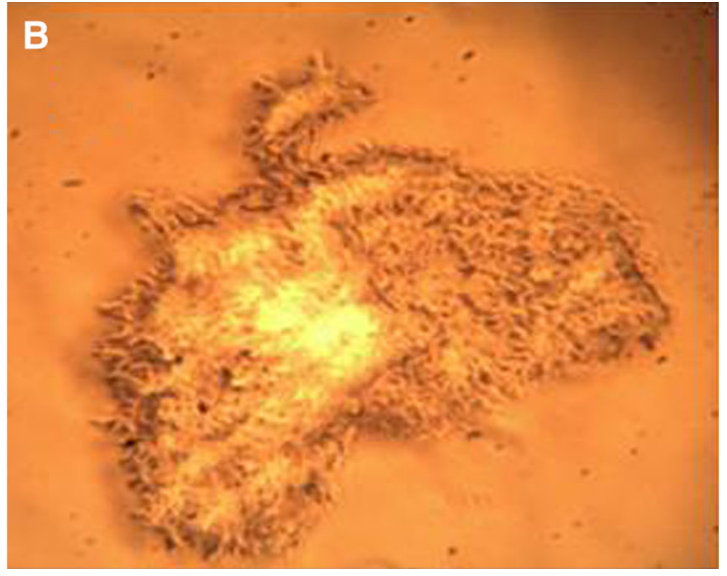

spherical shape of it, whereas on the $\mathbf{b}$ it is represented the picture of the condensed in ethanol solution opened-shape particle

solutions. For $200 \mu \mathrm{l}$ of DMSO, the percentile of bound allopurinol was equal to $110.04 \pm 10.0$, for $400-62.53 \pm 1.25$, for $800-31.26 \pm 5.63$ and for $2 \mathrm{ml}-0.5 \pm 0.75$; for water solution of allopurinol: for $\quad 200 \mu \mathrm{l}-70.03 \pm 40.02, \quad 400-57.52 \pm 11.26$, $800-13.76 \pm 0.63, \quad 2 \mathrm{ml}-6.0 \pm 0.0 \quad$ (Fig. 4). $p$ value in DMSO group was $<0.05$ (Kruskal-Wallis one-way ANOVA on ranks).

6. Stability experiments (Fig. 5).

Particles were more stable until 15 th $\mathrm{h}$ of incubation at $4{ }^{\circ} \mathrm{C}$ in DMSO solution rather than in water. The volume 


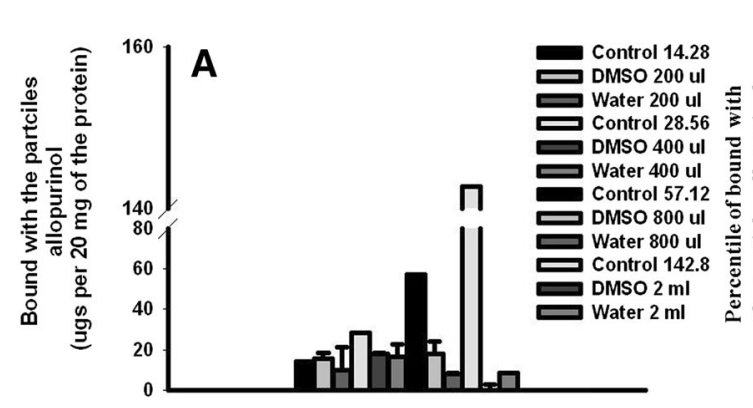

Fig. 4 Determination of bound with particle allopurinol quantity. PerkinElmer Junior spectrophotometer was used to estimate the quantity of the allopurinol in the samples. a Bound with the $20 \mathrm{mg}$ of the particles allopurinol. Allopurinol was added as a water solution or as a dissolved in DMSO compound in the volumes equal to 200, 400,

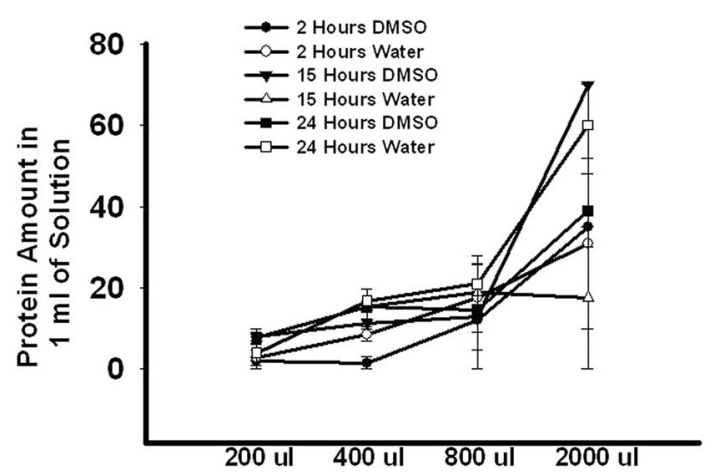

Fig. 5 Estimation of the particles stability over 24 h. Particles were suspended in water as well as in DMSO of different volumes (200, $400 \mu \mathrm{l}$ and 800 and $2 \mathrm{ml}$ ). Three time points were chosen $2 \mathrm{~h}, 15$ and $24 \mathrm{~h}$ of incubation. The suspensions were centrifugated, and the in supernatant the quantity of the proteins was assessed

of solution was also the defining factor for the stability experiments (larger volume was correlating with visualized less stability of the particles). The difference between 2-h incubation in $200 \mu \mathrm{l}$ of DMSO and $24 \mathrm{~h}$ was statistically significant $(2 \pm 0 ; 39 \pm 9 ; \quad p<$ 0.044153 ); between 2 and $15 \mathrm{~h}$ for $400-\mu \mathrm{l}$ volume in DMSO was also different in statistically significant way $(1.5 \pm 1,5$ vs $11.2 \pm 1.2 ; p<0.037052)$; between 2 and $24 \mathrm{~h}$ for $800 \mu \mathrm{l}(12 \pm 7.2$ vs $14 . \pm 0.6, p<0.00226$, DMSO groups). Similar trend of dissolution of the particles was noticed for water suspensions (Fig. 5).

\section{Discussion}

There are numerous publications describing the formation of the albumin particles, which are very useful for the treatment of cancer because of specific accumulation of the albumin and because of its receptors existence in cancer tissue. The experimental mixtures might contain albumin and

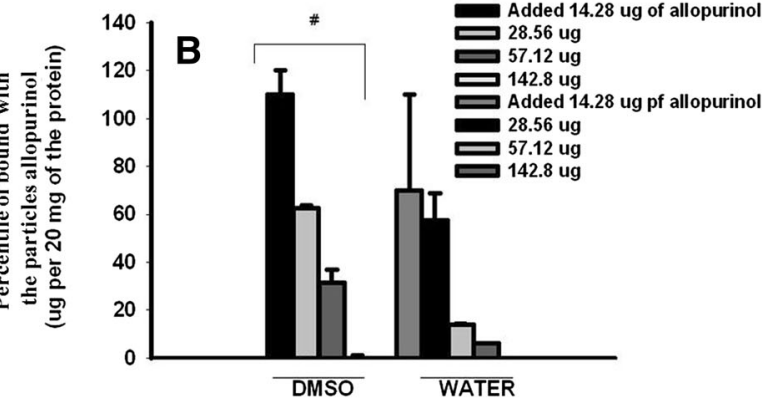

$800,2000 \mu \mathrm{l}(0.07 \mathrm{mg} / \mathrm{ml})$. Controls point to the concentration of the allopurinol as stock solutions of comparable with the samples volumes. b Effectiveness (\%) of allopurinol binding with the particles. The concentration of the equal volume stocks solution of the allopurinol was accounted as a $100 \%$

hypromellose acetate succinate [22], maleic anhydride molecules, resulting in disk-shaped particles, and BSA with $N^{\prime}, N^{\prime}$-dimethylacrylamide in water-in-oil and using ammonium persulfate as an initiating agent [23], resulting in formation of spheroid like 1-5 $\mu$ particle [24]. We have used GA as described in the study of Zhao D. [25]. In the first set of the experiments, we were trying to evaluate the quantity of GA which will be efficiently conjugating albumin and will be forming the most useful shaped particles. After the first set of the experiments, we concluded that $50 \mu \mathrm{l}$ of GA is the best quantity, which forms the smallest particles. During the second set of the experiments, it was found that the minimal quantity of the protein might force the formation of the small-size particle, and the number or quantity of the particles in these conditions was very high. Spheroid-type particles were chosen due to their large total surface which will be able to carry the highest concentration of the albumin. The allopurinol was not specifically conjugated with the albumin particles to guarantee the free possible penetration of the medicines in the capillaries after surface-surface tight interaction of them with the endothelial cells to provoke highly efficient penetration of allopurinol as the medicine the treatment of ischemic stroke.

Also, we noticed that the binding of allopurinol with the particles is much more effective, when DMSO and nonwater solutions are used. It might be suggested that the higher effectiveness is based on abilities of allopurinol solubility in water vs DMSO. Also, from Fig. 4 it is clear that the binding process is much more effective in the smaller volumes. This process is reflecting stability of the particles in the solution. This means that in $24 \mathrm{~h}$ in aqueous solution or in the blood, these particles might be dissolved, which is the positive ability for these formulations and evidences about spontaneous biodegradable ability with the increase in albumin in plasma (Fig. 5). Increased plasma albumin by itself might serve as a wonderful compound in stroke treatment as the oncotic pressure-determining agent, 
preventing diffusion of the water from blood via disrupted hematoencephalic barrier into the brain parenchyma.

In our studies, we have shown that inhibition of $\mathrm{XO}$ by its classical inhibitor might initiate regenerative processes in neuronal cell culture by the inhibition of purine catabolism by feedback mechanism as well as by the inhibition of XOgenerated ROS [26-28]. On the other hand, we also propose the utility of such albumin particles for the treatment of stroke. Albumin is the compound, which might be useful in the near future for the treatment of stroke [29] as an antihemorrhagic and anti-ischemic agent. It is in clinical trials [30].

A method for the generation 1- to 5-micron spheroid particles has been adopted to assess the allopurinol quantity in the coated particles, which might be useful in the future for the treatment of ischemic stroke.

Acknowledgments The study was supported by the basic financing of National Academy of Science of Armenia.

Open Access This article is distributed under the terms of the Creative Commons Attribution 4.0 International License (http://crea tivecommons.org/licenses/by/4.0/), which permits unrestricted use, distribution, and reproduction in any medium, provided you give appropriate credit to the original author(s) and the source, provide a link to the Creative Commons license, and indicate if changes were made.

\section{References}

1. Peters, T.: All about albumin: biochemistry, genetics and medical applications. Academic Press Limited, San Diego (1996)

2. Sudlow, G., Birkett, D.J., et al.: Further characterization of specific drug binding sites on human serum albumin. Mol. Pharmacol. 12, 1052-1061 (1976)

3. de Wolf, F.A., Brett, G.M.: Ligand-binding proteins: their potential for application in systems for controlled delivery and uptake of ligands. Pharmacol. Rev. 52(2), 207-236 (2000)

4. Curry, S.: Lessons from the crystallographic analysis of small molecule binding to human serum albumin. Drug Metab. Pharmacokinet. 24, 342-357 (2009)

5. Curry, S., Brick, P., et al.: Fatty acid binding to human serum albumin: new insights from crystallographic studies. Biochim. Biophys. Acta 1441, 131-140 (1999)

6. Bhattacharya, A.A., Grüne, T., et al.: Crystallographic analysis reveals common modes of binding of medium and long-chain fatty acids to human serum albumin. J. Mol. Biol. 303, 721-732 (2000)

7. Sudlow, G., Birkett, D.J., et al.: The characterization of two specific drug binding sites on human serum albumin. Mol. Pharmacol. 11, 824-832 (1975)

8. Zunszain, P.A., Ghuman, J., et al.: Crystallographic analysis of human serum albumin complexed with 4Z, 15E-bilirubin-IX $\alpha$. J. Mol. Biol. 381(2), 394-406 (2008)

9. Kragh-Hansen, U.: Molecular aspects of ligand binding to serum albumin. Pharmacol. Rev. 33, 17-53 (1981)

10. Evans, T.W.: Review article: albumin as a drug-biological effects of albumin unrelated to oncotic pressure. Aliment. Pharmacol. Ther. 16(Suppl. 5), 6-11 (2002)

11. Elzoghby, A.O., Samy, W.M., et al.: Protein-based nanocarriers as promising drug and gene delivery systems. J. Control. Release 161, 38-49 (2012)
12. Nitta, S.K., Numata, K.: Biopolymer-based nanoparticles for drug/gene delivery and tissue engineering. Int. J. Mol. Sci. 14(1), 1629-1654 (2013)

13. Dawson, J., Quinn, T.J., et al.: The effect of allopurinol on the cerebral vasculature of patients with subcortical stroke; a randomized trial. Br. J. Clin. Pharmacol. 68(5), 662-668 (2009)

14. Taheraghdam, A.A., Sharifipour, E., et al.: Allopurinol as a preventive contrivance after acute ischemic stroke in patients with a high level of serum uric acid: a randomized, controlled trial. Med. Princ. Pract. 23(2), 134-139 (2014)

15. Kim, T.H., Jiang, H.H., et al.: Preparation and characterization of Apo2L/TNF-related apoptosis-inducing ligand-loaded human serum albumin nanoparticles with improved stability and tumor distribution. J. Pharm. Sci. 100(2), 482-491 (2011)

16. Sebak, S., Mirzaei, M., et al.: Human serum albumin nanoparticles as an efficient noscapine drug delivery system for potential use in breast cancer: preparation and in vitro analysis. Int. J. Nanomed. 5, 525-532 (2010)

17. Zhang, L.K., Hou, S.X., et al.: Preparation, characterization, and in vivo evaluation of mitoxantrone-loaded, folate-conjugated albumin nanoparticles. Arch. Pharm. Res. 33(8), 1193-1198 (2010)

18. Li, F.Q., Su, H., et al.: Preparation and characterization of sodium ferulate entrapped bovine serum albumin nanoparticles for liver targeting. Int. J. Pharm. 349(1-2), 274-282 (2008)

19. Zhao, L., Su, R., et al.: Preparation of biocompatible heat-labile enterotoxin subunit B-bovine serum albumin nanoparticles for improving tumor-targeted drug delivery via heat-labile enterotoxin subunit B mediation. Int. J. Nanomed. 9, 2149-2156 (2014)

20. Suprun, P.P.: Improvement of existing and elaboration of new technic of iodometric quantitative determination of xanthine derivatives by formation of periodide. Farm Zh. 26(5), 65-69 (1971)

21. Arzamaszev, A.P.: Pharmchemistry course, 2nd edn, p. 319. Medicine, Moscow (1995)

22. Lee, K.N., Ye, Y., et al.: Formulation, pharmacokinetics and biodistribution of ofloxacin-loaded albumin microparticles and nanoparticles. J. Microencapsul. 28(5), 363-369 (2011)

23. Sitta, D.L., Guilherme, M.R., et al.: Covalent albumin microparticles as an adjuvant for production of mucosal vaccines against hepatitis B. Biomacromolecules 14(9), 3231-3237 (2013)

24. Sitta, D.L., Guilherme, M.R., et al.: Drug release mechanisms of chemically cross-linked albumin microparticles: effect of the matrix erosion. Colloids Surf. B Biointerfaces 122, 404-413 (2014)

25. Zhao, D., Zhao, X., et al.: Preparation, characterization, and in vitro targeted delivery of folate-decorated paclitaxel-loaded bovine serum albumin nanoparticles. Int. J. Nanomed. 5, 669-677 (2010)

26. Danielyan, K.E.: Dependence of cells survival from xanthine oxidase and dihydopyrimidine dehydrogenase correlative activities in human brain derived cell culture. Cent. Nerv. Syst. Agents Med. Chem. 13(2), 108-113 (2013)

27. Danielyan, K.E., Chailyan, S.G.: Xanthine dehydrogenase inhibition stimulates growth and development of human brain derived cells. Am. J. Med. Biol. Res. 1(4), 95-98 (2013)

28. Gyongyan, S.A., Manucharyan, T.G., et al.: Xanthine oxidoreductase is a key enzyme of purine catabolism regulation. Electron. J. Nat. Sci. 5(2), 17 (2013)

29. Belayev, L., Saul, I., et al.: Albumin treatment reduces neurological deficit and protects blood-brain barrier integrity after acute intracortical hematoma in the rat. Stroke 36(2), 326-331 (2005)

30. Hill, M.D., Martin, R.H., et al.: The albumin in acute stroke part 1 trial: an exploratory efficacy analysis. ALIAS investigators; neurological emergencies treatment trials network. Stroke 42(6), 1621-1625 (2011) 